\title{
Artemisia annua: A New Version of a Traditional Tea under Randomized, Controlled Clinical Trial for the Treatment of Malaria
}

\author{
Pedro Melillo de Magalhães ${ }^{1}$, Glyn Mara Figueira' ${ }^{1}$, José Maria de Souza ${ }^{2}$, \\ Ana Maria Revorêdo Ventura², Maria Deise de Oliveira Ohnishi' ${ }^{2}$, Darci Rodrigues da Silva², \\ Luiz Alberto Gonçalves Lobo², Fabrício Bezerra Eleres², Rosana Libonati ${ }^{3}$, Merlin Willcox ${ }^{4}$, \\ Eloisa Cavassani Pimentel ${ }^{5}$
}

\author{
${ }^{1}$ University of Campinas, Campinas, Brazil \\ ${ }^{2}$ Evandro Chagas Institute, Belém, Brazil \\ ${ }^{3}$ Federal University of Pará, Belém, Brazil \\ ${ }^{4}$ University of Oxford (Research Initiative for Traditional Antimalarial Methods), Oxford, UK \\ ${ }^{5}$ Campinas Municipal Centre of Health, Campinas, Brazil \\ Email: pedro@cpqba.unicamp.br
}

How to cite this paper: de Magalhães, P.M., Figueira, G.M., de Souza, J.M., Ventura, A.M.R., de Oliveira Ohnishi, M.D., da Silva, D.R., Lobo, L.A.G., Eleres, F.B., Libonati, R., Willcox, M. and Pimentel, E.C. (2016) Artemisia annua: A New Version of a Traditional Tea under Randomized, Controlled Clinical Trial for the Treatment of Malaria. Advances in Bioscience and Biotechnology, 7, 545-563.

http://dx.doi.org/10.4236/abb.2016.712049

Received: October 10, 2016

Accepted: December 9, 2016

Published: December 12, 2016

Copyright $\odot 2016$ by authors and Scientific Research Publishing Inc. This work is licensed under the Creative Commons Attribution International License (CC BY 4.0).

http://creativecommons.org/licenses/by/4.0/ (c) (i) Open Access

\begin{abstract}
Introduction: The traditional antimalarial tea Artemisia annua, indicated for centuries in China to treat fevers, is again arousing interest for the treatment of malaria due to improvements attained in the plant composition by a few Institutions throughout the world, including the State University of Campinas (UNICAMP), Brazil, increasing its principal component by more than 100 times as from standard varieties, giving $1 \%$ in artemisinin and an expressive biomass yield such as 2 tons of dried leaves/hectare. Clinical trials carried out with this material in African countries have proven its therapeutic potential for a new generation of Artemisia tea in the treatment of falciparum malaria. In addition to artemisinin, recent studies have identified and quantified other compounds present in the crude extract and characterized their contributions to the anti-malarial efficacy, including their action against chloroquineresistant strains. The majority of the clinical trials carried out with Artemisia tea in African countries have shown that the control of the parasitaemia is efficient in the initial treatment period, but few trials have followed the patients up to the $28^{\text {th }}$ day. This first clinical trial carried out in Brazil with the $A$. annua infusion, after toxicological trials that defined the safety of this form of medication. Methods: The therapeutic efficacy of the tea was measured in patients with falciparum malaria over 28 days, comparing it with the current first-line treatment namely artemether-lumefantrine $\left(\right.$ Coartem $\left.^{\circledast}\right)$. The trial was carried out in controlled groups according to offi-
\end{abstract}


cial protocol approved by the National Ethics in Research Committee (CONEP: 77/ 2011) and a rigorous control of the 17 patients with non-serious cases of falciparum malaria, recruited in the following three municipalities of the State of Pará, Brazil: Tucuruí, Goianésia do Pará and Anajás. The tea group received the infusion prepared in the proportion of $1.25 \mathrm{~g}$ of dry leaves of the variety CPQBA with $1 \%$ artemisinin for $250 \mathrm{~mL}$ of just boiled water, taken every 6 hours for 7 days, giving a total of approximately $175 \mathrm{mg}$ of artemisinin, whilst the artemether-lumefantrine (Coartem ${ }^{\circledast}$ ) group received a total of $525 \mathrm{mg}$ of artemisinin equivalent to artemether. Results: The parasitaemia by the tea treatment became negative in the first days, even though it was administered with a dose that was one third of the recommended dose of artemisinin. However, as in the case artemisinin or artesunate monotherapies, $57.1 \%$ of the patients treated with the $A$. annua tea presented type I resistance, with a return of the parasitaemia around the $14^{\text {th }}$ or $21^{\text {st }}$ day. The other patients in the tea group showed type II/III resistance without manifestation of any serious signs or symptoms. In these cases, according to the protocol, the patients were redirected for treatment with artemether-lumefantrin $\left(\right.$ Coartem $\left.^{\oplus}\right)$ with subsequent negativity of the parisitaemia. Discussion: The fact that the efficacy of the tea with $1 / 3$ of the dose of artemisinin was similar to that of the full dose of this medication infers that other compounds present in the crude extract, probably flavonoids, had contributed to the negativity of the parasitaemia at the start of the treatment. Considering that the positive control group, where the compound derived from artemisinin (artemether) was associated with another antimalarial agent (lumefantrine), presented excellent efficacy throughout the entire control of the cure, future trials with the $A$. annua tea should use the same strategy of association with another antimalarial agent, preferably from $A$. annua itself, in order to extend its therapeutic action during the whole control period. The Artemisia annua tea in the form standardized and used in this research, should not substitute the most efficient treatment, but could be considered as an emergency therapeutic resource in the first hours of symptoms as a function of its availability, anti-inflammatory action and lack of side effects. Other regimes and standardizations deserve investigation, mainly those with a high content of arteannuin $B$, as occurs in the initial cultivation phase.

\section{Keywords}

Malaria, Herbal Medicine, Artemisia annua, Clinical Trial

\section{Introduction}

The species Artemisia annua L., Asteraceae, native of Asia, has been indicated since ancient times as an anti-fever agent, and was described by Ge Hong in 340 A.D. in his Manual of Prescriptions for Emergences [1] (Zhou Hou Bei Ji Fang). The traditional form of preparation shows variations, both infusions of the leaves in hot water and their immersion in water at room temperature being cited. In the 1970s, based on prescriptions from Traditional Chinese Medicine, the Chinese researcher Tu Youyou 
(Nobel Prize for Medicine, 2015) isolated the molecule of artemisinin from $A$. annua leaves [2], and this became the safest and most efficient chemical structure discovered to date to combat the parasite Plasmodium, causer of malaria. Artemisinin is a sesquiterpene lactone containing an endoperoxide group (Figure 1), which is decisive in the control of the protozoa Plasmodium, a parasite which infects the red blood corpuscles.

As from this discovery, the pharmaceutical industry dedicated itself to the development of antimalarial agents containing pure artemisinin extracted from $A$. annua leaves, and eventually developed more stable and efficient artemisinin derivatives by semisynthesis, such as sodium artesunate and artemether. The search for the base molecule, artemisinin, led to genetic selection projects aimed at increasing the content of the active principle in the leaves and per area, with a view to satisfying the demand and improving industrial extraction, isolation and purification yields. Throughout the world, the following two institutions were pioneers in this agronomical development, promoting, in addition to hybrids and populations 100 times richer in artemisinin in relation to the original populations, the necessary technology for the cultivation and postharvest processing of the species: Mediplant in Switzerland and the State University of Campinas (UNICAMP) in Brazil. In addition, some important research scientists, such as John Laughlin (Australia), Jorge Ferreira (USA), Pierre Lutgen (Luxemburg) and James Simon (USA) also acted in the agro-technology of $A$. annua resulting in important contributions. More recently the University of York (UK) developed plants even richer in artemisinin. Although A. annua is the only herbal raw material for the production of artemisinin, significant technological advances have been made by the University of California, Amyris, USA for the total synthesis of the molecule as from artemisininic acid, which is produced by yeasts [3]. However, it has been estimated that there will always be a market for both sources of the molecule, since the plant manufactures other compounds of interest in malarial therapy.

In the use of artemisinin or its derivatives in malaria therapy, which are also efficient in the control of $P$. falciparum strains resistant to the antimalarial agents previously used, the fact that the parasite could also acquire resistance to artemisinin is of concern.
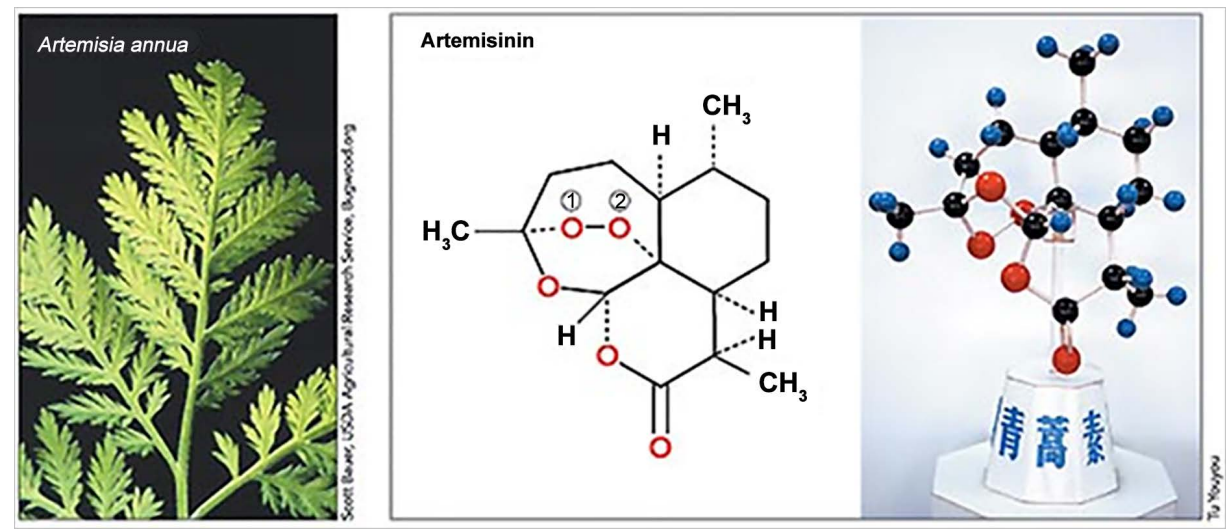

Figure 1. Artemisinin: the most efficient molecule against $P$. falciparum. 
This could occur either by way of mutant genes that could acquire tolerance, or by a population constituted of artemisinin-resistant genotypes resulting from selection by the antimalarial agent itself. These two processes are present in malaria therapy, and inexorably promote, with time, a resistant population to a determined antimalarial agent. However, in the case of artemisinin, these processes occur with greater difficulty due to the mode of action of the molecule, which acts in a physicochemical way on the Plasmodium. In fact, artemisinin is one of those rare molecules found in nature that have an endo-peroxide bridge in their structure with a double bond between oxygens, which, when broken, produces a sort of "micro-explosion" capable of destroying the membrane of a red blood corpuscle (RBC) parasitized by Plasmodium. However, this "micro-explosion" that destroys both the $\mathrm{RBC}$ and the parasite, only occurs around infected $\mathrm{RBC}$, since these produce a reducing ambient on their surface which causes the double bond of the peroxide bridge of the artemisinin to break. It is an extremely selective mechanism, since the artemisinin in the blood stream will get together with all the $\mathrm{RBC}$ due to its affinity for iron, but will only destroy infected ones. As a consequence, the Plasmodium will also be destroyed since its membrane will be broken. However even with this mechanism in place there is the possibility that some infected RBC will not be destroyed, either because they are resistant to the physicochemical effect of artemisinin, or because they are surrounded by other healthy RBC that neutralize the reducing ambient, forming agglomerates known as "rosettes". Thus in the case of monotherapy with artemisinin or its derivatives the threat exists of the development of populations resistant to these pure antimalarial agents due to the selective process and not because they acquired resistance. The action of artemisinin or even its derivative Artesunate when used in monotherapy, was shown not to be efficient throughout the whole cure period, that is, the phenomenon of recrudescence occurred, signaling the danger of resistance, since tolerant or resistant genes could be present in the parasitic population. However this is not the only hypothesis for the occurrence of recrudescence, since the phenomenon can occur by other means, such as: the artemisinin was already degraded before acting against all the parasites; that some parasites were protected by the action of the antimalarial agent in microcirculation cells, or; by red blood cells adhered one to another forming structures known as rosettes, protecting infected $\mathrm{RBC}$ and preventing the action of artemisinin or its derivatives on its membranes, as mentioned earlier. As a function of this behavior and aiming to prolong the longevity of the best antimalarial therapeutic agent to date, the World Health Organization (WHO) preconized the use of $A$. annua antimalarial preparations always in association with other antimalarial agents with distinct action modes and/or with a longer half-life in the blood stream [4]. Thus the product Coartem (arthemeter + lumefantrine) and other associated antimalarial agents (ACTs-Artesunate Combined Treatment) have been on the market for more than 10 years, with high efficacy and safety.

Although the registered ACTs show known efficiency and safety, they are not always available in the endemic regions of poor countries where mortality is still to the order of 1 million people per year. In fact, for the treatment of malaria, apart from efficiency 
and safety, availability of the medication is fundamental, which, for its part, involves adhesion, distribution, price, and governmental policies.

These fundamental characteristics for the efficacy of an antimalarial agent are present in the $A$. annua tea, including the aspect of association, since the artemisinin is extracted from the trichomes of the leaves together with other molecules that have antimalarial action, or, at least, specific synergic action with the artemisinin by various mechanisms, such as: anti-inflammatory action [5]; more efficient transport through the intestinal barrier [6]; or an increase in susceptibility of Plasmodium by way of arteannuin B. However, one cannot affirm if the $A$. annua tea favors or avoids the process of resistance to artemisinin. Nevertheless, is reinforced the hypothesis that the synergism between artemisinin and certain other compounds present in the $A$. annua tea make the development of resistance difficult, even though the total amount of artemisinin administered in the treatment with the infusion is below that that was recommended for the treatment with pure artemisinin. During the process for the commercial development of antimalarial agents from isolated and purified artemisinin, the other substances produced by the plant are discarded. However, recent studies have reevaluated the composition of the crude extract, this time using selected plants, and identified and quantified the principal components and their antimalarial, antagonistic or synergistic action with artemisinin. Some compounds, such as arteannuin B, show important synergism with the artemisinin, improving its efficiency, including that against chloroquine-resistant $P$. falciparum strains [7] [8]. The molecule arteannuin B is the precursor of artemisinin.

The present clinical study englobes earlier multidisciplinary research carried out over 25 years with the species at CPQBA-UNICAMP, since it evaluates the maximum finality, which is: the therapeutic efficacy of the $A$. annua infusion prepared with the leaves of an artemisinin-rich genotype and in combination with other compounds of interest in the plant. When a medication reaches the phase of clinical research, be it synthetic or of natural origin, this opens the opportunity for a complete evaluation, including even adjustments to the dosage, formulation and therapeutic regime. It is hence a decisive step in the improvement of its use, and in this case, represents an important step for other plants of the Brazilian flora to be evaluated. Without doubt, the tea obtained from the variety UNICAMP-1, with $1 \%$ artemisinin, is highly distinct from that prepared with the leaves from wild plants, where the artemisinin content was to the order of $0.005 \%$ $0.01 \%$.

The idea to test the efficiency of the tea from an improved $A$. annua plant was born in 1995, when Magalhães et al. showed that the infusion extracted approximately $60 \%$ of the total artemisinin from the leaves [9]. The first institution to vulgarize this therapeutic form was the German NGO ANAMED-Action for Natural Medicine, as from promising results obtained in various African countries since 1996. Subsequent clinical trials carried out in African countries demonstrated the therapeutic potential of Artemisia tea, despite the occurrence of some cases of recrudescence which can be partly explained by the short half-life of artemisinin in the plasma [10] [11] [12]. Apart from 
ANAMED, other European non-governmental organizations acting in African countries started recommending the use of $A$. annua infusions, gathering together successful cases of the treatment of falciparum malaria with the tea made from $A$. annua leaves, including: the ICEI-Istituto Cooperazione Economica Internazionale, and IFBV-Techniques Durables et Solidaires. In recent years, renewed scientific interest has been shown in the pharmacological potential of the tea made from genotypes of $A$. annua containing high artemisinin contents, as related to malaria [13] [14] [15] and other infectious-parasitic diseases, such as toxoplasmosis, schistosomiasis and also anticancer action [16] [17] [18] besides anti-HIV [19]. The anti-inflammatory action detected by Prof. Yves-Jaques Schneider's group at the University of Louvain-la-Neuve (Belgium) and the facilitated passage of artemisinin through the intestinal barrier when $A$. annua tea is taken, open new horizons, demonstrating that compounds of important pharmacological action for malaria, such as the anti-inflammatory action, are present in the tea. However, many of these earlier results were not conclusive with respect to the real efficacy of the tea from new $A$. annua genotypes in the treatment of human malaria, since they did not follow the official WHO protocol for the control of the patients as done in this clinical research. It seemed to us to be fundamental for scientists involved in therapies for malaria, to carry out a clinical trial within the criteria accepted by the scientific community, and rigorously following the guidelines of the World Health Organization for the evaluation of antimalarial medication. Thus we did not start from the pretext that the tea could be more efficient than the therapeutic option currently in first place, Coartem, but aimed to evaluate the efficiency of the infusion in relation to Coartem. In agreement with this posture was the fact that there was absolutely no commercial interest in the $A$. annua tea.

\section{Material and Methods}

\subsection{Raw Material}

The $A$. annua variety CPQBA-UNICAMP was cultivated in Paulínia-SP-Brazil, Lat. $22^{\circ} 48^{\prime} 02.38^{\prime \prime}$, long. $47^{\circ} 06^{\prime} 43.10^{\prime \prime} \mathrm{W}$, alt. $612 \mathrm{~m}$, irrigated by spraying and the weeds controlled by manual weeding until the disappearance of the lines between the plants due to their development. The soil in the experimental area is classified as typical clayey eutroferric red latosol. The seedlings were formed in sleeves under screening with 50\% interception in the period between August and September in the years 2009, 2010 and 2011 , and cultivated in the field for approximately 4 months as from October of the respective years, with a spacing of $0.5 \times 1.0 \mathrm{~m}$. Cultivation was carried out during the 3 years of the project aimed at always treating the recruited patients with new leaves, within the stipulated shelf life of 12 months. With the objective of always obtaining raw material with the highest possible artemisinin content, harvesting was done in the state preceding flowering and only the top third of the plant was harvested, resulting in a artemisinin content between $1.0 \%$ and $1.1 \%$ [20].The leaves were sun-dried with periodic turning over, followed by manual separation of the stalks and thick twigs. All the batches of $A$. annua used in the project were cultivated at CPQBA-UNICAMP follow- 
ing the same cultivation and drying procedures and their artemisinin content remained in the range from $0.9 \%$ to $1.1 \%$ in relation to the dry weight of the leaves harvested (Figure 2).Thus the final raw material consisted only of leaves, and was sent to FiocruzFar manguinhos, where it was ground and packed into individual sachets of aluminized paper, each containing exactly $1.25 \mathrm{~g}$ of dried ground leaves (Figure 3 ).

\subsection{Protocol and FCTC}

The proposal to carry out the clinical phase II trial in Brazil was approved in the Pronex-Rede Malaria note in 2009, the study being financed exclusively by the Brazilian Government Organ CNPq-MS and FAPESP, as well as the support of the following institutions: State University of Campinas, Farmanguinhos, Campinas Health Secretariat, the Tucuruí, Anajás and Goianésia hospitals in the State of Pará, Brazil, and, principally, the Evandro Chagas Institute in Belém do Pará, Brazil. The protocol for the clinical trial, the Free and Clarified Term of Consent (FCTC) and the questionnaire used in the

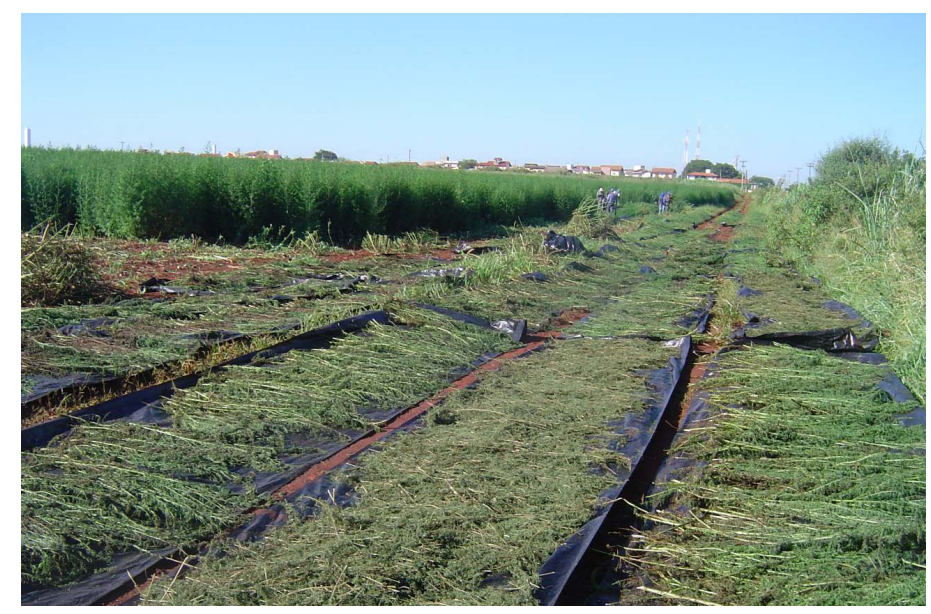

Figure 2. Manual harvest and early drier of $A$. annua leaves at CPQBAUNICAMP.

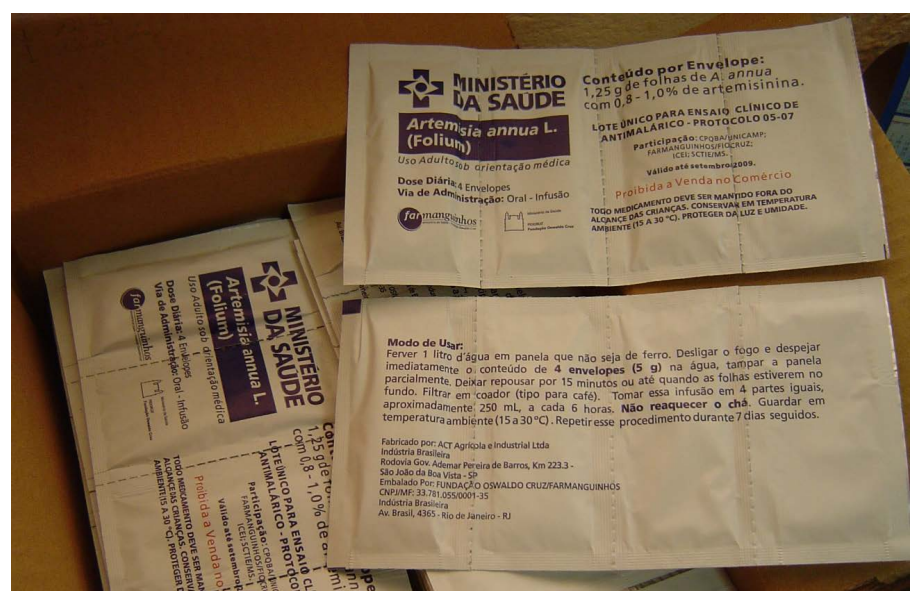

Figure 3. Aluminized sachets, exclusively prepared to this clinical trial, each containing $1.25 \mathrm{~g}$ dried $A$. annua leaves with 1, $0 \%$ artemisinin. 
patient interviews, were all approved by the Ethics in Research Committee of the Evandro Chagas Institute and by the National Committee for Ethics in Research (CONEP), n 77/2011.

The clinical research protocol and the Free and Clarified Term of Consent were elaborated rigorously following the recommendations found in the WHO guidelines, number 05.1 of 2005, entitled "Assessment and monitoring of antimalarial drug efficacy for the treatment of uncomplicated falciparum malaria", with adaptations and CONSORT statement [21]. Based on previous research carried out by Mueller et al. (2000) and Mueller et al. (2004) which showed the efficacy of $A$. annua tea to be about $74 \%$ in the therapy of falciparum malaria, the number of patients necessary was calculated as being two unilateral samples of 61 patients. This would give statistical certainty to demonstrate differences with $80 \%$ of power at $5 \%$ of significance, calculated by the proportion test. Considering an estimated a rate of loss of $10 \%$ during the procedure, the number of patients in each sample (Coartem and tea) was defined as 70 (Total number of patients $=140$ ). The research was directed at uncomplicated cases of malaria in patients infected by $P$. falciparum. Recovery of the patients with Coartem was planned in the case of any eventual therapeutic failure or resistance of any type, as also specific medical attention in these cases.

\subsection{Inclusion and Exclusion Criteria}

The patients were recruited according to the following inclusion criteria: age between 18 and 50, weight between 40 and $80 \mathrm{Kg}$, parasitaemia below 50,000 asexual forms $/ \mathrm{mm}^{3}$ of blood with no dysfunction of vital organs, absence of other serious concomitant diseases, and a declaration of consent. The exclusion criteria were defined according to the presence of, at least, one of the following danger signals: incapacity to eat or drink, more than 2 episodes of vomiting in the last 24 hours, unconsciousness, showing the incapacity to sit down or stand up without help, parasitaemia above $50,000 / \mathrm{mm}^{3}$ blood, or presenting severe malaria as defined by the WHO criteria: Severe malaria is characterized by presenting: alterations in respiratory rate (respiratory rate above 20 and the use of accessory muscles), jaundice (clinically diagnosed), clinically severe anemia, $\mathrm{Hb}$ $<5 \mathrm{~g} / \mathrm{dl}$, or evidence of paleness, alterations in respiratory function, tachycardia, high blood pressure during the exams, presenting spontaneous mouth or gum bleeding, sys-

tolic blood pressure below $80 \mathrm{~mm} \mathrm{Hg}$ with signs of low peripheral perfusion, and being incapable of knowing where he/she is or give an appropriate verbal response to a painful stimulus or suffer a generalized convulsion in the previous 30 minutes. The exclusion criteria also include: being pregnant as determined by the B-HCG-urine test, being allergic to artemisinin and/or lumefantrine, and having used an antimalarial agent in the last 20 days.

\subsection{Recruitment and Logistics (Epidemiological Survey-External Monitoring; Adjustment of Criteria, Home Care, Changing Municipalities and Changes in Tea Preparation)}

The location chosen for installation of the research was defined considering: recent 
epidemiological history, profile of the patients (avoiding gold digging regions since these patients are difficult to control), the infrastructure of the local hospital, and access to allow assessment by health professionals from the Evandro Chagas Institute in BelémPA-Brazil. The first region chosen according to these criteria was the municipality of Tucuruí-PA. By way of complex logistics, the following were installed in the Tucuruí Regional Hospital for use in the project: a microscope, a nurse, and a health agent/ driver, all from the Evandro Chagas Institute (IEC). Periodic visits were also made by doctors from the IEC, responsible for all the medical-scientific activities of the project and by the project coordinator and external assessors. The municipality of Tucuruí has 4 public health clinics, and, on agreeing to take part in the research, the patients recruited in these clinics signed the FCTC and were sent to the Tucuruí Regional Hospital where they were hospitalized throughout the whole treatment ( 7 days) independent of the randomly chosen treatment. Control of the cure and parasitaemia was carried out after 14, 21 and 28 days. In addition to parasitaemia, the control of the patients in the two groups evaluated the biochemical parameters on days D-0, D-3, D-7, D-21 and D-28. The laboratory analyses were: CBC (red blood corpuscles, platelets, leucocytes, segmented cells, lymphocytes, eosinophils, monocytes and reticulocytes); coagulation (bleeding time, coagulation time, coagulum retraction, prothrombin time, prothrombin activity and thromboplastin time); aspartate aminotransferase (AST), alanine aminotransferase (ALT), urea, creatine, uric acid, glycaemia, high sensitive C-reactive protein (Hs-CRP), blood group and urine (volume).

\subsection{Preparation of the Treatments and Follow-Up of the Patients}

In the majority of cases the patients were diagnosed, treated and controlled in the $\mathrm{Tu}$ curuí Hospital, where they were hospitalized for 7 days. In the other recruitment locations, Goianésia do Pará and Anajás, the initial diagnosis was done in the hospital, whereas treatment and control were done at home. A temperature-controlled room was made available in each of the hospitals (Tucuruí, Goianésia do Pará and Anajás) for storage of the $A$. annua tea sachets, for the documentation of the patients, and, for preparation of the treatments according to the experimental protocol. Every 6 hours for 7 days, $300 \mathrm{~mL}$ of filtered water was heated to boiling point in a stainless steel recipient, the recipient removed from the heat, and the dry $A$. annua leaves added, stirred briefly and then left to rest for 15 minutes in a covered cup. The tea was then filtered and given to the patient to drink. The members of the control group, who were treated with Coartem, followed the dosage and therapeutic regime of this medication, and were also hospitalized for 7 days for a comparative accompaniment involving the use of the same laboratory analyses for the two groups, as well as using the same accommodation and catering program. However, the patients recruited in Anajás received their $A$. annua tea prepared in a different way: after reaching boiling point, the leaves were added and boiled for 2 minutes before removing from the heat and leaving to rest at room temperature for 15 minutes. This alteration was based on information that the amount of artemisinin extracted was significantly greater when the leaves were boiled for $2 \mathrm{mi}$ - 
nutes [22].

\section{Results}

\subsection{Raw Material}

The batches were submitted to an analysis for microbial contamination during storage, but were all considered innocuous [23]. This infers that the high camphor content of the essential oil and the low moisture content of the stored leaves inhibited microbial development.

\subsection{Therapeutic Efficacy}

The main parameter followed in the therapeutic treatment of malaria was the monitoring of parasitaemia, together with a clinical follow-up of the patient.

\subsubsection{Parasitaemia Results}

As shown in Table 1, parasite clearance by Coartem was excellent and all the patients showed negative results between the second and third days of treatment, with no occurrence of recrudescence up to the 28th day. This justifies the comparative proposal of this research, using the most efficient medication as the positive control. The reduced number of patients is explained by the early closure of recruitment due to the successive occurrence of unsatisfactory efficiency of the treatment with Artemisia tea as shown in Table 2.

Despite the fact that $50 \%$ of the patients showed negative results for parasitaemia in the first days of treatment with Artemisia tea, recrudescence occurred after about two or three weeks, when the patients were rescued using appropriate medicine, in this case Coartem. In the case of therapeutic failure, R2 and R3, this behavior coincided with the change in region and modification of the tea-making procedure. The change in region to Anajás on the Isle of Marajó was necessary due to the low endemic level of cases of falciparum malaria in Tucuruí and Goianésia do Pará, which made recruitment difficult

Table 1. Parasitaemia of patients infected with $P$. falciparum and treated with Coartem.

\begin{tabular}{lcccc}
\hline Coartem & \multicolumn{4}{c}{ Parasitaemia against 200 leucocytes } \\
\hline D0 & D1 & D2 & D3 \\
\hline Patient 1 & 85 & 0 & 0 & 0 \\
Patient 2 & 15 & 0 & 0 & 0 \\
Patient 3 & 8 & 2 & 0 & 0 \\
Patient 4 & 76 & 22 & 0 & 0 \\
Patient 5 & 122 & 25 & 0 & 0 \\
Patient 6 & 20 & 3 & 0 & 0 \\
Patient 7 & 253 & 99 & 14 & 0 \\
Patient 8 & 294 & 177 & 2 & 0 \\
\hline
\end{tabular}


Table 2. Control of patients treated with an infusion of Artemisina annua var. CPQBA.

\begin{tabular}{cccccccc}
\hline Tea & \multicolumn{7}{c}{ Parasitaemia against 200 leucocytes } \\
\hline & D0 & D1 & D2 & D3 & D4 & Recrudescence & Resistance \\
\hline Patient 1 & 19 & 168 & & & & R3 \\
Patient 2 & 177 & 307 & 304 & & & R3 \\
Patient 3 & 10 & 6 & 4 & 0 & 0 & D10 & R1 \\
Patient 4 & 52 & 21 & 1 & 0 & 0 & D14 \\
Patient 5 & 149 & 104 & 35 & 9 & 0 & D21 & R1 \\
Patient 6 & 105 & 48 & 13 & 2 & 0 & D14 & R1 \\
Patient 7 & 18 & 224 & & & & R3 \\
Patient 8 & 92 & 38 & 105 & & & R2 \\
\hline
\end{tabular}

within the designated project time. In order to maintain the same quality of the Artemisia leaves with an artemisinin content of $1.1 \%$, new cultures were developed at CPQBAUNICAMP, such that the material was never more than 6 months old. The whole harvesting and post-harvest processing procedure was rigorously standardized for the 3 cultures. The change in tea-making procedure was due to a publication by Kooy \& Verpoorte (2011), who showed that more artemisinin was extracted when the infusion was prepared by boiling the leaves for exactly two minutes. The possibility remains that this procedure could have degraded other substances, such as arteannuin B, and, in this way, have reduced the parasite control efficiency. Although the phenomenon of recrudescence could have been expected in the tea group, considering the relatively short half-life of the compounds in the blood stream, one should also consider the possibility that the Plasmodium falciparum from the Isle of Marajó could have been genetically more sensitive to artemisinin in relation to the same parasite found in Goianésia do Pará and in Tucuruí.

\subsubsection{Bio-Clinical Analyses}

The laboratory analyses were: CBC (red blood corpuscles, platelets, leucocytes, segmented cells, lymphocytes, eosinophils, monocytes and reticulocytes); coagulation (bleeding time, coagulation time, coagulum retraction, prothrombin time, prothrombin activity and thromboplastin time); aspartate aminotransferase (AST), alanine aminotransferase (ALT), urea, creatine, uric acid, glycaemia, high sensitive C-reactive protein (Hs-CRP), blood group and urine (volume). Despite the limited number of patients, there were significant differences for the parameters: coagulation time, bleeding time and prothrombin time. Figures 4-6 show the means obtained for these parameters in the groups treated with Artemisia annua tea and with Coartem.

These data show that in the tea group there was less alteration in the coagulation factors with respect to the bleeding, coagulation and prothrombin times. 


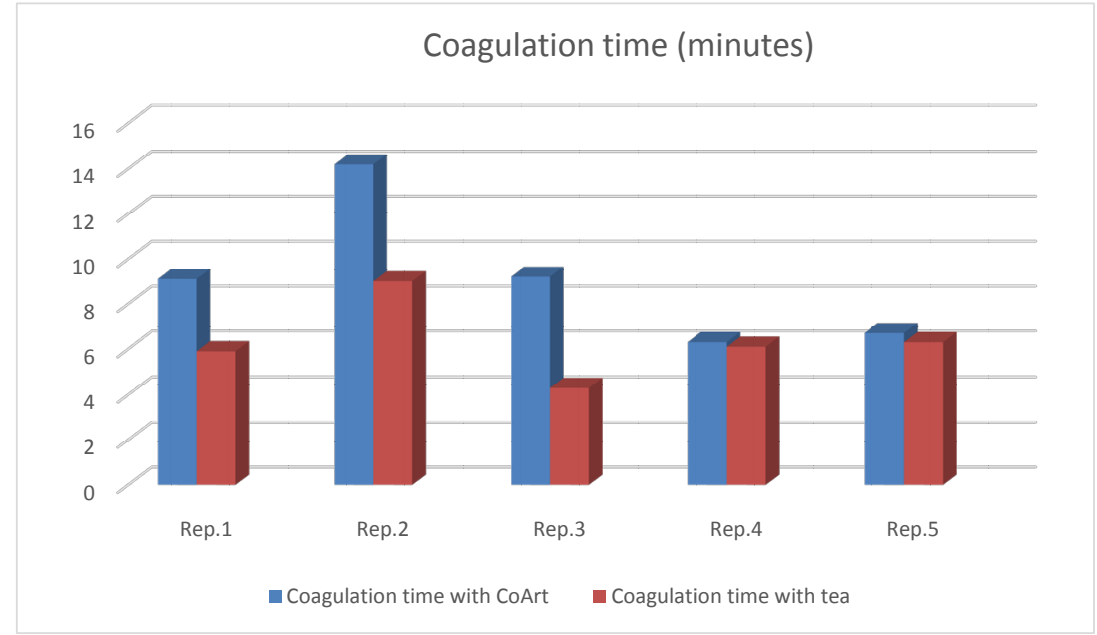

Figure 4. Coagulation time (minutes).

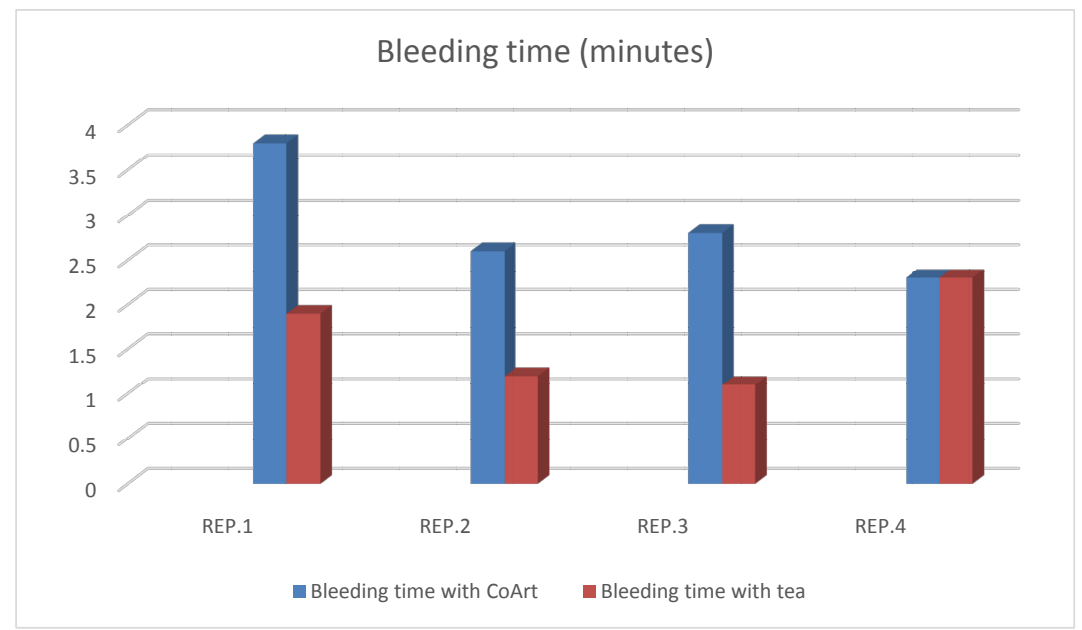

Figure 5. Bleeding time (minutes).

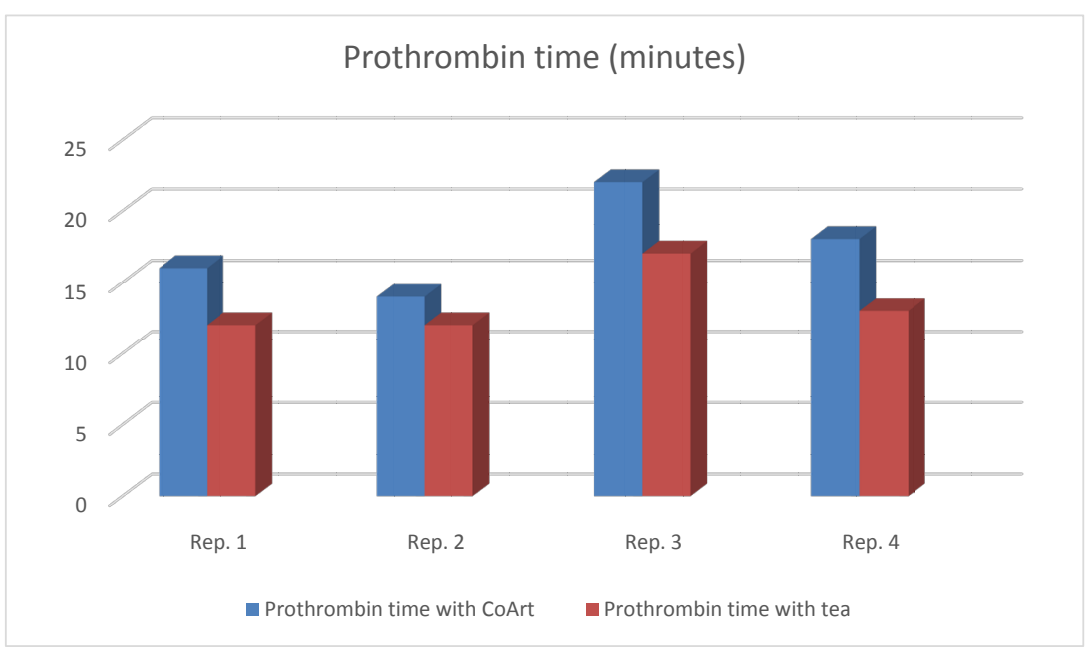

Figure 6. Prothrombin time (minutes). 
The normal times are: for bleeding, between 2 - 8 minutes (Ivy test), for prothrombin, between $80 \%-100 \%$ or from 11 to 14.6 seconds, and for coagulation, from 4 to 10 min. It can be seen that, in general, the bleeding and coagulation times were within the normal ranges for both groups, although the tea-taking patients showed shorter times than those in the Coartem group. With respect to the prothrombin time, the Coartem patients showed a time that was more prolonged than normal. In fact, the longer the prothrombin time, the smaller its concentration in the blood. This parameter evaluates the extrinsic coagulation route, and one of the factors that lead to its alteration is consumption coagulopathy (disseminated intravascular coagulation). The bleeding time evaluates the efficiency of the platelets. The coagulation time evaluates the intrinsic coagulation route, and, if altered, confirms defects in the coagulation factors. The patients in the Coartem group presented a mean value for initial parasitaemia much higher and a mean platelets count much lower than the tea group, which, despite not being significantly different, could have contributed to these results.

\subsubsection{Interviews with Tea Group Patients after Treatment}

After treatment, the patients who took the Artemisia tea reported that they felt good throughout the entire treatment and that the bitter taste was tolerable. Some of them referred to this taste as "the more bitter, the better", showing an empiric sensitivity concerning the drugs traditionally recommended for malaria therapy. No patient reported feeling sick or vomiting. Even those who showed recrudescence did not present symptoms of the disease, and questioned the need for a rescue treatment with Coartem. Nevertheless, according to Brazilian policies for the control of falciparum malaria, with a view to reducing transmission, cases of recrudescence are treated with Coartem for the total control of the infection, even if, as in these cases, they were asymptomatic (In some African countries a patient is not obliged to treat asymptomatic infections since they consider that this immune response of the monocytes, even if temporary, can be an advantage for the host. Nevertheless, he is collaborating with the maintenance of the focus of the disease and its transmission. Currently the reduction in $P$. vivax parasitaemia is considered in Brazil, treating the cases of resistance even when the infection is asymptomatic. This procedure is essential in the context of eliminating malaria). In many African countries the therapy of promoting a reduction in parasitaemia until the symptoms are eliminated is accepted in the control of malaria. However, this does not necessarily signify that the infection has been controlled, since asymptomatic recrudescence could have occurred. It can be said that the asymptomatic patient has acquired a degree of immunity, and has the right to continue the treatment required to completely control the parasitaemia, or otherwise. As a consequence, such an asymptomatic patient collaborates with an increase in transmission if bitten by the vector. In Brazil, the policy of treating malaria preconizes the total control of parasitaemia, aimed exactly at reducing transmission. For this reason, and also to assure that no parasite remains that could promote the selection of a population resistant to the antimalarial agents, the control of the cure of malaria falciparum should demonstrate that the parasitaemia remains negative up to the 28 th day. 
Despite the low number of patients recruited, a higher value was observed for the monocytes in the group treated with $A$. annua tea (non-statistically significant difference).

\section{Discussion}

Important advances and changes in the paradigms were made in this research, which included work led by UNICAMP with the support of various partner entities over 28 years, in addition to reaching the specific objective of knowing the efficiency of Artemisia annua tea in malarial therapy, after innovating the raw material. Four large segments, united in this proposal, can be highlighted: agronomy, chemistry, biology and medicine. The studies in the ambience of agronomy concentrated on the genetic improvement of the plant, adapting it to the inter-tropical and tropical conditions, where malaria occurs. By monitoring the chemical composition and the use of biological trials, the plant can be improved with respect to its yield of active substances (content and biomass), not only of artemisinin, but also of molecules that take part in the biological actions under investigation, such as the case of the flavonoids. Knowledge of the plant physiology and also of the parameters related to post-harvest processing involving the drying, storage and making of the tea, were decisive points in the standardization of the raw material. However, it was the carrying out of this clinical research together with the accompanying of the data in the literature that allowed for an integrated understanding of the various segments. As a result, we now know the current efficiency of this form of medication and the direction in which to carry out adjustments aimed at improvements, as occurs in the development of all pharmaceutical products. The innovation of developing medication to treat malaria as from a plant opens the possibility for new studies using the same model, with other vegetable species which have the advantage of counting with traditional use, and hence with strong adhesion by the populations who live in endemic areas. Traditional medication can have new versions and amplify its benefits if its development is based on the same integrated scientific arrangement used in this research, making the most of what Nature produced. This research fits into the context of a clinical study of medicinal plants from the Brazilian flora, with the potential to improve the treatment of malaria in the form of simple preparations, albeit safe and efficient, which are well accepted by the local population [24].

Another innovative aspect, intrinsic to the use of the crude extract, was the evaluation of the "active complex", in contrast to conventional studies focused on a single "active principal". In fact, the plant produces an arsenal of substances with specific functions, frequently synergetic with the main active molecule. What could have been lost with the use of the pure substance? One must consider there are no reports of resistance to the tea or of any side effects, and that the tea contains various substances many of which are antimalarial, representing an association of antimalarial substances thus complying with the strategy of not using the pure substance, however efficient it may be.

Although this research was initially considered polemic, since it dealt with a disease 
of risk and generated concern about the development of resistance, the proposed project guaranteed that it would be carried out in a controlled group, following the official protocol approved by CONEP, and hence received financial support from the main Brazilian governmental organs as an expression of its scientific legitimacy and without conflicts of interests.

Two facts should be considered with respect to the concern about the development of resistance. However, first one should clarify the possible types of resistance in order to give support to the discussion. The following types of resistance exist: 1) resistance caused by the selection of a Plasmodium population, that is, some individuals resistant to the drug remain as the only survivors and multiply, forming a resistant population, 2) some individuals were not exposed to the drug, either because they were in microcirculation or because they were protected by other RBC (rosettes, cyto-adhesion) and multiply after the drug has already degraded, and 3) by mutation of Plasmodium which starts interacting with the drug. With respect to the latter case, which deserves more attention, the fact that the tea was used for more than a thousand years in Asia in the traditional way, without any reports of resistance, is encouraging. With the current knowledge concerning the action mechanism of artemisinin in destroying Plasmodium, which is predominantly physical, breaking the endo-peroxide bridges, there is a strong argument justifying the absence of resistance to Artemisia tea and the anti-malarial drugs based on artemisinin. However, the second fact that collaborates with the understanding that Artemisia tea does not favor the development of resistance, and, to the contrary, reverts this process, are the findings of Suberu et al. (2013), who showed that the compound Arteannuin B, present in the tea, interacts with the artemisinin for the chloroquine resistant strains. This fact also justifies the evaluation of Artemisia tea for the therapeutic treatment of malaria caused by $P$. vivax, which currently represents from $5 \%$ to $8 \%$ of the resistance attributed to chloroquine-resistant parasites. In addition, with the information of Nair et al. [25] that the leaves harvested after only one month of cultivation in the field have 2.9 times more Arteannuin B than artemisinin, one can prepare a tea from a blend of two harvests, or even start treatment with a batch with a high artemisinin content (harvested after 4 months) and then continue with another batch richer in Arteannuin B (harvested after 2 months).

The fact that $A$. annua tea promotes a drastic reduction in the initial parasitaemia is a great, but not excellent, result, making it necessary to search for adjustments in the medication and regime. The sooner the parasetemiais quelled, the better, since one more day of infection represents one more day of transmission by the vector. In its present stage of development, the $A$. annua tea could be an important resource for first aid.

Due to the consecutive occurrence of recrudescence in the patients recruited into the $A$. annua tea group and rigorously following the protocol without any modifications that could improve the efficiency of this treatment, the clinical research had to be terminated before reaching the predicted number of patients. One should therefore consider the results in order to provide recommendations and directions for future research. 
Thus we understand that an adequate direction for the next actions with $A$. annua tea should involve: 1) not encouraging its use as a substitute for the treatment with artemether + lumefantrin since, although the patients showed an excellent clinical response, recrudescence (type I resistance), or no decrease or even an increase in parasitaemia (type II/type III resistance) did occur; and 2) evaluate the efficacy of the $A$. annua tea in association with other antimalaric species or compounds from a plant like Arteannuin $\mathrm{B}$, maintaining the principal of simple and available medication; 3 ) evaluate the efficacy of the $A$. annua tea in a new therapeutic regime, including a dose on day14, aimed at avoiding recrudescence; 4) during the follow up evaluate eventual inflammatory alterations (PCR-US) in the patients and also the monocyte content when used concomitantly with artemether + lumefantrin; and 5) evaluate the efficacy of the $A$. annua tea when associated with the conventional malaria vivax treatment. 6) Evaluate the nutritional status of the patients in both groups, and also the blood test $(\mathrm{CBC})$ parameters.

\section{Conclusions and Perspectives}

As in the case of artemisinin or artesunate monotherapies [26] [27] [28], 57.1\% of the patients treated with the $A$. annua tea presented type I resistance, with a return of the parasitaemia around the 14th or 21 st days of the cure control.

This fact demonstrates that the Artemisia tea is still not a final product, but on the other hand should not be considered inefficient and hence banned, since it is a product under development and its current efficiency is already important for the initial fight against Plasmodium, in the context of the first therapeutic actions. It is evident that its current efficiency does not allow it to substitute the official medication, but it could eventually be considered as part of the therapy, principally because the official medication is not always immediately available. In fact, the ease of access and acceptance of the Artemisia tea by the population, added to the absence of collateral effects, characterizes an important drug resource. Efforts to improve its efficiency by way of associations and adjustments to the regime are part of the development of this important therapeutic tool against malaria. Direction of future research: administer the dose on the 14th day; take it associated with the conventional medication focusing on the anti-inflammatory property (controlling the exacerbated inflammation); take a mixture or concomitantly with other species, for example: Pogostemon cablin, Chenopodium ambrosioides, Bidens pilosa, Curcuma longa, Baccharis dracunculifolia (nerolidol); test the $A$. annua tea in vivax malaria using the same principals used here.

This was the first clinical Brazilian research with phyto-medication directed at the treatment of malaria. In addition to the scientific results, the teams were capacitated and specific points in the research with the raw material identified, mainly with respect to standardization.

\section{Acknowledgements}

The authors thank State University of Campinas, Evandro Chagas Institute (Belém), FIOCRUZ-farmanguinhos, Campinas Health Secretariat, Tucuruí Regional Hospital, 
Goianésia do Pará Hospital, and Anajás Hospital.

\section{Author's Note}

During this research, the present author was infected with malaria by $P$. knowlesi in the region of Kota Kinabalu-Malasia, and was cured using Artemisia annua tea. This result is not part of the data presented.

\section{Support}

This work was supported by CNPq-MS, FAPESP, and FAEPEX.

\section{References}

[1] Ge, H. (Dongjin Dynasty) (2000) Zhou Hou Bei Ji Fang. Tianjin Science \& Technology Press, Tianjin.

[2] Tu, Y.Y. (1999) The Development of New Antimalarial Drugs: Qinghaosu and DihydroQinghaosu. Chinese Medical Journal, 112, 976-977.

[3] Paddon, C.J., Westfall, P.J., Pitera, D.J., Benjamin, K., Fisher, K., Mcphee, D., Leavell, M. D., Tai, A., Main, A., Eng, D., Polichuk, D.R., Teoh, K.H., Reed, D.W., Treynor, T., Lenihan, J., Jiang, H., Fleck, M., Bajad, S., Dang, G., Dengrove, D., Diola, D., Dorin, G., Ellens, K.W., Fickes, S., Galazzo, J., Gaucher, S.P., Geistlinger, T., Henry, R., Hepp, M., Horning, T., Iqbal, T., Kizer, L., Lieu, B., Melis, D., Moss, N., Regentin, R., Secrest, S., Tsuruta, H., Vazquez, R., Westblade, L.F., Xu, L., Yu, M., Zhang, Y., Zhao, L., Lievense, J., Covello, P.S., Keasling, J.D., Reiling, K.K., Renninger, N.S. and Newman, J.D. (2013) High-Level SemiSynthetic Production of the Potent Antimalarial Artemisinin. Nature, 496, 528-532. https://doi.org/10.1038/nature12051

[4] WHO (2003) Assessment and Monitoring of Antimalarial Drug Efficacy for the Treatment of Uncomplicated Falciparum Malaria.

[5] Magalhães, P., Dupont, I., Hendrickx, A., Joly, A., Raas, T., Dessy, S., Sergent, T. and Schneider, Y.-J. (2012) Anti-Inflammatory Effect and Modulation of Cytochrome P450 Activities by Artemisia annua Tea Infusions in Human Intestinal Caco-2 Cells. Food Chemistry, 134, 864-871. https://doi.org/10.1016/j.foodchem.2012.02.195

[6] Magalhaes, P.M., Schneider, Y.-J., Dupont, I., Frederich, M. and Jonville, M.-C. (2010) New Strategy to in Vitro Evaluation of Antimalarial Drugs Demonstrate Advantage of Artemisia annua Crude Extract on Antimalarial Effect by Its Tools to Cross the Intestinal Barrier. In: XII Reunião Nacional de Pesquisa em Malária, Sociedade Brasileira de Imunologia, Ouro Preto, 114.

[7] Suberu, J.O., Gorka, A.P., Jacobs, L., Roepe, P.D., Sullivan, N., Barker, G.C. and Lapkin, A.A. (2013) Anti-Plasmodial Polyvalent Interactions in Artemisia annua L. Aqueous Extract-Possible Synergistic and Resistance Mechanisms. PLOS ONE, 8, e80790. https://doi.org/10.1371/journal.pone.0080790

[8] Elfawala, M.A., Towlerb, M.J., Reichc, N.G., Weathers P.J. and Richa, S.M. (2014) Dried Whole-Plant Artemisia annua Slows Evolution of Malaria Drug Resistance and Overcomes Resistance to Artemisinin. PNAS, 112, 821-826. https://doi.org/10.1073/pnas.1413127112

[9] Magalhaes, P.M., Debrunner, N. and Delabays, N. (2003) Aqueous Extracts of Artemisia annua L. International Conference on Malaria: Currant Status and Future Trends, Bangkok, 16-19 February 2003.

[10] Mueller, M.S., Karhagomba, I.B., Hirt, H.M. and Wemakor, E. (2000) The Potential of Ar- 
temisia annua L. as a Locally Produced Remedy for Malaria in the Tropics: Agricultural, Chemical and Clinical Aspects. Journal of Ethnopharmacology, 73, 487-493.

https://doi.org/10.1016/S0378-8741(00)00289-0

[11] Mueller, M.S., Runyambo, N., Wagner, I., Borrmann, S., Dietz, K. and Heide, L. (2004) Randomized Controlled Trial of a Traditional Preparation of Artemisia annua L. (Annual Wormwood) in the Treatment of Malaria. Transactions of the Royal Society of Tropical Medicine and Hygiene, 98, 318-321.

https://doi.org/10.1016/j.trstmh.2003.09.001

[12] Rath, K., Taxis, K., Walz, G., Gleiter, C.H., Li, S.M. and Heide, L. (2004) Pharmacokinetic Study of Artemisinin after Oral Intake of a Traditional Preparation of Artemisia annua L. (Annual Wormwood). The American Journal of Tropical Medicine and Hygiene, 70, 128132.

[13] Graz, B., Willcox, M., BerthÉ, D., Ardiet, D.L., Falquet, J., Diallo, D. and Giani, S. (2015) Home Treatments Alone or Mixed with Modern Treatments for Malaria in Finkolo AC, South Mali: Reported Use, Outcomes and Changes over 10 Years. Transactions of the Royal Society of Tropical Medicine and Hygiene, 109, 209-213. https://doi.org/10.1093/trstmh/tru181

[14] Willcox, M. (2011) Improved Traditional Phytomedicines in Current Use for the Clinical Treatment of Malaria. Planta Medica, 77, 662-671. https://doi.org/10.1055/s-0030-1250548

[15] Willcox, M., Burton, S., Oyweka, R., Namyalo, R., Challand, S. and Lindsey, K. (2011) Evaluation and Pharmacovigilance of Projects Promoting Cultivation and Local Use of $A r$ temisia annua for Malaria. Malaria Journal, 10, 84. https://doi.org/10.1186/1475-2875-10-84

[16] Oliveira, T.C., Oliveira Silva, D.A. and Rostkowska, C. (2009) Artemisia annua L. on Susceptibility to Infection in Experimental Models in Vitro and in Vivo. Experimental Parasitology, 122, 233-241. https://doi.org/10.1016/j.exppara.2009.04.010

[17] Frezza, T.F., Oliveira, C.N.F., Banin, T.M., Rehder,V., Boaventura Júnior, S. and Allegretti, S.M. (2013) Tegumentary Changes in Two Different Strains of Schistosoma mansoni Treated with Artemisinin and Artesunic Acid. Revista de Patologia Tropical (Online), 42, 309-321.

[18] Efferth, T., Herrmann, F., Tahrani, A. and Wink, M. (2011) Cytotoxic Activity of Secondary Metabolites Derived from Artemisia annua L. towards Cancer Cells in Comparison to Its Designated Active Constituent Artemisinin. Phytomedicine, 18, 959-969. https://doi.org/10.1016/j.phymed.2011.06.008

[19] Lubbe, A., Seibert, I., Klimkait, T. and Van Der Kooy, F. (2012) Ethnopharmacology in Overdrive: The Remarkable Anti-HIV Activity of Artemisia annua. Journal of Ethnopharmacology, 141, 854-859. https://doi.org/10.1016/j.jep.2012.03.024

[20] Sousa, I.M.O., Rodrigues, R.A.F., Queiroz, N.C.A., Alves, A., Celeghini, R.M.S., Nobrega, A.B., Magalhaes, P.M. and Foglio, M.A. (2009) Comparison of Sachet (Aluminium) Package with Conventional Bags Preserving Artemisinin of Artemisia annua L. 2nd Brazilian Conference of Natural Products, São Pedro, SP, Brasil, 2009, 1-2.

[21] Gagnier, J.J., Boon, H., Rochon, P., Moher, D., Barnes, J. and Bombardier, C. (2006) Reporting Randomized, Controlled Trials of Herbal Interventions: An Elaborated Consort Statement. Annals of Internal Medicine, 144, 364-367. https://doi.org/10.7326/0003-4819-144-5-200603070-00013

[22] Van Der Kooy, F. and Verpoorte, R. (2011) The Content of Artemisinin in the Artemisia annua Tea Infusion. Planta Medica, 77, 1754-1756. https://doi.org/10.1055/s-0030-1271065

[23] Cervezan, T.C.M., Broetto, F., Figueira, G.M., Rabonato, A.C. and Souza, I.M.O. (2013) 
Avaliação fitoquímica e microbiológica da espécie Artemisia annua L., submetida a tratamentos de armazenamento e condições de ambiente. Revista Energia na Agricultura, 28, 270-276.

[24] Weathers, P.J., Towler, M., Hassanali, A., Lutgen, P. and Engeu, P.O. (2014) Dried-Leaf Artemisia annua: A Practical Malaria Therapeutic for Developing Countries? World Journal of Pharmacology, 3, 39-55. https://doi.org/10.5497/wjp.v3.i4.39

[25] Nair, M.S. and Basile, D.V. (1993) Bioconversion of Arteannuin B to Artemisinin. Journal of Natural Products, 56, 1559-1566. https://doi.org/10.1021/np50099a015

[26] Alin, M.H., Ashton, M., Kihamia, C.M., Mtey, G.J.B. and Bjorkman, A. (1996) Clinical Efficacy and Pharmacokinetics of Artemisinin Monotherapy and in Combination with Mefloquine in Patients with Falciparum Malária. British Journal of Clinical Pharmacology, 41, 587-592. https://doi.org/10.1046/j.1365-2125.1996.35116.x

[27] Giao, P.T., Binh, T.Q., Kager, P.A., Long, H.P., Van Thang, N., Van Nam, N. and De Vries, P.J. (2001) Artemisinin for Treatment of Uncomplicated Falciparum Malaria: Is There a Place for Monotherapy? The American Journal of Tropical Medicine and Hygiene, 65, 690695.

[28] Teuscher, F., Gatton, M.L, Chen, N., Peters, J., Kyle, D.E. and Cheng, Q. (2010) Artemisinin-Induced Dormancy in Plasmodium falciparum: Duration, Recovery Rates, and Implications in Treatment Failure. The Journal of Infectious Diseases, 202, 1362-1368. https://doi.org/10.1086/656476

\section{Submit or recommend next manuscript to SCIRP and we will provide best service} for you:

Accepting pre-submission inquiries through Email, Facebook, LinkedIn, Twitter, etc.

A wide selection of journals (inclusive of 9 subjects, more than 200 journals)

Providing 24-hour high-quality service

User-friendly online submission system

Fair and swift peer-review system

Efficient typesetting and proofreading procedure

Display of the result of downloads and visits, as well as the number of cited articles

Maximum dissemination of your research work

Submit your manuscript at: http://papersubmission.scirp.org/

Or contact abb@scirp.org 